\title{
Finite-Horizon Estimation of Randomly Occurring Faults for A Class of Nonlinear Time-Varying Systems
}

\author{
Hongli Dong a,b,*, Zidong Wang ${ }^{c}$, Steven X. Ding ${ }^{b}$, Huijun Gao ${ }^{\mathrm{d}}$ \\ ${ }^{a}$ College of Electrical and Information Engineering, Northeast Petroleum University, Daqing 163318, China. \\ ${ }^{\mathrm{b}}$ Institute for Automatic Control and Complex Systems, University of Duisburg-Essen, 47057, Germany. \\ ${ }^{\mathrm{c}}$ Department of Computer Science, Brunel University, Uxbridge, Middlesex, UB8 3PH, U.K. \\ ${ }^{\mathrm{d}}$ Research Institute of Intelligent Control and Systems, Harbin Institute of Technology, Harbin 150001, China.
}

\begin{abstract}
This paper is concerned with the finite-horizon estimation problem of randomly occurring faults for a class of nonlinear systems whose parameters are all time-varying. The faults are assumed to occur in a random way governed by two sets of Bernoulli distributed white sequences. The stochastic nonlinearities entering the system are described by statistical means that can cover several classes of well-studied nonlinearities. The aim of the problem is to estimate the random faults, over a finite horizon, such that the influence from the exogenous disturbances onto the estimation errors is attenuated at the given level quantified by a $H_{\infty}$-norm in the mean square sense. By using the completing squares method and stochastic analysis techniques, necessary and sufficient conditions are established for the existence of the desired finite-horizon $H_{\infty}$ fault estimator whose parameters are then obtained by solving coupled backward recursive Riccati difference equations (RDEs). A simulation example is utilized to illustrate the effectiveness of the proposed fault estimation method.
\end{abstract}

Key words: Fault estimation; Randomly occurring faults; Time-varying systems; Nonlinear stochastic systems; Recursive Riccati difference equations.

\section{Introduction}

Over the past decades, fault detection has been an active research topic because of the growing demand for higher performance, higher safety and stricter reliability standards in a highly automated industrial system, and a variety of important results have been reported in the literature, see e.g. [2-4, 7, 14, 16, 20, 22, 25, 28]. Fault detection itself, however, is insufficient to guarantee the reliability especially for systems with critical safety requirements such as aircraft flight control systems. Fault estimation, on the other hand, is capable of providing accurate size and shape of the fault, thereby helping reconstruct the fault signals. As such, fault estimation is further needed for the purpose of active fault tolerant control. Up to date, considerable research attention has been devoted to the theoretical research on the fault estimation problem, and a great number of fault estimation approaches have been proposed in existing literature, see e.g. $[6,11-13,15,17,19,27,30]$ and the references therein. For example, in $[11,12,19,29]$, the problems of fault estimation have been investigated by applying adaptive

\footnotetext{
* This work was supported in part by the National Natural Science Foundation of China under Grants 61329301, 61134009, 61273156, 61333012 and 61374127, the Scientific and Technology Research Foundation of Heilongjiang Education Department of China under Grant 12541061, the Engineering and Physical Sciences Research Council (EPSRC) of the U.K., the Royal Society of the U.K., and the Alexander von Humboldt Foundation of Germany.

* Corresponding author.

Email addresses: shiningdhl@gmail.com (Hongli Dong), Zidong. Wang@brunel.ac.uk (Zidong Wang).
}

fault diagnosis observers that can improve the rapidity of fault estimation. In $[13,27]$, the sliding mode observerbased fault estimation method has been presented to realize fault reconstruction. A fault estimator has been developed in [6] based on a descriptor system formulation for the sensor fault estimation problem, which can estimate the states and the sensor fault signal simultaneously.

In reality, almost all real-time systems have certain timevarying parameters and, for such time-varying systems, one would be more interested in their transient performances over a finite period than the traditional steadystate behaviors over the infinite-horizon. When it comes to the fault estimation issue, finite-horizon fault estimators for time-varying systems are of particular engineering significance especially when the noise inputs are nonstationary $[17,30]$. It should be pointed out that, compared to the fruitful results on infinite-horizon fault estimation problems for time-invariant systems, the corresponding results on finite-horizon fault estimation problems are still in its early stages due probably to the mathematical/computational complexities. Very recently, in [17], the finite-horizon $H_{\infty}$ fault estimation problem has been investigated for a class of uncertain linear discrete time-varying systems with known inputs, where all the estimator parameters have been derived simultaneously in terms of an explicit solution to a matrix equation. In [30], with the aid of the parity-space method, a fault estimator for linear discrete time-varying systems has been designed. Furthermore, the $H_{\infty}$ fault estimation problem has been investigated in [31] by using the Kreinspace theory. However, in almost all existing results concerning finite-horizon fault estimation problems, it has 
been implicitly assumed that the system is linear and the occurred fault signals are instantaneous.

Recently, as the network scale increases, the randomly occurring phenomena (ROP) have become an emerging research topic that has drawn some initial research attention. ROP refer to those phenomena that appear intermittently in a random way based on certain probability law [5]. If not properly coped with, the ROP would unavoidably deteriorate the operating efficiency or even cause the instability of the plant. So far, a series of results have been reported in the literature concerning the filtering and control problems in the presence of ROP, for example, randomly occurring nonlinearities [4,18], randomly occurring uncertainties [10], randomly occurring sensor delays [4] and randomly occurring sensor saturations [23]. Nevertheless, an important class of networkinduced phenomena, namely, randomly occurring faults (ROFs), have been largely overlooked despite their practical significance in networked control systems. In fact, due to bandwidth limitation of the shared links and unpredictable variation of the network conditions, severe packet loss, data collision or data quantization could be regarded as a kind of faults when the reliability becomes a concern. The network-induced faults may also stem from issues concerning the aging, disturbances, electromagnetic interference, temporary failure of the sensors or actuators. Such faults typically occur in a probabilistic way and it would make practical sense to consider the ROFs where the occurrence probability can be estimated via statistical tests. So far, the ROF-based fault estimation problem has not received adequate research attention yet, not to mention the case when nonlinear and time-varying natures are also taken into account. It is, therefore, the main purpose of this paper to shorten such a gap by addressing the finite-horizon estimation problem of ROFs for a class of nonlinear discrete timevarying systems.

Motivated by the above discussions, in this paper, we aim to investigate the finite-horizon estimation problem of randomly occurring faults for a class of nonlinear systems whose parameters are all time-varying. The main contributions of this paper are highlighted as follows. 1 ) The concept of randomly occurring faults is proposed to better reflect the random nature of the occurrences of sensor and actuator failures induced by networks. 2) Necessary and sufficient conditions are obtained for the existence of the desired fault estimator by applying the completing squares method and stochastic analysis technology. 3) A novel coupled Riccati difference equation approach is developed to solve the finite-horizon $H_{\infty}$ fault estimation problem.

The rest of this paper is outlined as follows. In Section 2 , the discrete time-varying nonlinear stochastic system with randomly occurring faults is introduced and the problem under consideration is formulated. In Section 3, the design problem of finite-horizon $H_{\infty}$ fault estimators is solved and a simulation example is given in Section 4 to demonstrate the main results obtained. Finally, we conclude the paper in Section 5 .

Notation The notation used here is fairly standard except where otherwise stated. $\mathbb{R}^{n}$ and $\mathbb{R}^{n \times m}$ denote, respectively, the $n$-dimensional Euclidean space and the set of all $n \times m$ real matrices. $l_{2}[0, \infty)$ is the space of square summable sequences. The notation $X \geq Y$ (respectively, $X>Y$ ), where $X$ and $Y$ are real symmetric matrices, means that $X-Y$ is positive semi-definite (respectively, positive definite). $M^{T}$ represents the transpose of the matrix $M$ and $M^{\dagger} \in \mathbb{R}^{n \times m}$ denotes the Moore-Penrose pseudo inverse of $M \in \mathbb{R}^{m \times n}$. We may fix a probability space $(\Omega, \mathscr{F}$, Prob), where Prob, the probability measure, has total mass $1 . \mathbb{E}\{x\}$ stands for the expectation of the stochastic variable $x$ with respect to the given probability measure Prob. 0 and $I$ denote, respectively, the zero matrix of compatible dimensions and the identity matrix of compatible dimensions. $\operatorname{diag}\{\cdots\}$ stands for a block-diagonal matrix. $\operatorname{tr}(\mathrm{A})$ represents the trace of a matrix $A$. Matrices, if not explicitly specified, are assumed to have compatible dimensions.

\section{Problem Formulation}

Consider the following class of discrete time-varying nonlinear stochastic systems defined on $k \in[0, N]$ :

$$
\left\{\begin{aligned}
x(k+1)= & A(k) x(k)+g(k, x(k))+B_{d}(k) d(k) \\
& +\alpha_{1}(k) B_{f}(k) f(k) \\
y(k)= & C(k) x(k)+h(k, x(k))+\alpha_{2}(k) D_{f}(k) f(k) \\
& +D_{d}(k) v(k) \\
x(0)= & x_{0}
\end{aligned}\right.
$$

where $x(k) \in \mathbb{R}^{n_{x}}$ represents the state vector; $y(k)^{(1)}$ $\mathbb{R}^{n_{y}}$ is the measurement signal; $d(k) \in \mathbb{R}^{n_{w}}, v(k) \in \mathbb{R}^{n_{v}}$ and $f(k) \in \mathbb{R}^{n_{l}}$ are, respectively, the disturbance input, the measurement noises, and the fault signal. Moreover, it is assumed that $d(k), v(k)$ and $f(k)$ belong to $l_{2}[0, \infty)$; and $x_{0}$ is an initial value that is unknown. $A(k), B_{f}(k)$, $B_{d}(k), C(k), D_{f}(k)$ and $D_{d}(k)$ are known, real, timevarying matrices with appropriate dimensions.

We assume that the functions $g(k, x(k))$ and $h(k, x(k))$ with $g(k, 0)=0$ and $h(k, 0)=0$ are stochastic nonlinear functions described by their statistical characteristics as follows

$$
\begin{gathered}
\mathbb{E}\left\{\left[\begin{array}{l}
g(k, x(k)) \\
h(k, x(k))
\end{array}\right] \mid x(k)\right\}=0, \\
\mathbb{E}\left\{\left[\begin{array}{l}
g(k, x(k)) \\
h(k, x(k))
\end{array}\right]\left[g^{\mathrm{T}}(j, x(j)) h^{\mathrm{T}}(j, x(j))\right] \mid x(k)\right\}=0, \\
k \neq j
\end{gathered}
$$

and

$$
\begin{aligned}
& \mathbb{E}\left\{\left[\begin{array}{l}
g(k, x(k)) \\
h(k, x(k))
\end{array}\right]\left[g^{\mathrm{T}}(k, x(k)) h^{\mathrm{T}}(k, x(k))\right] \mid x(k)\right\} \\
= & \sum_{i=1}^{q} \pi_{i} \pi_{i}^{\mathrm{T}} \mathbb{E}\left\{x^{\mathrm{T}}(k) \Gamma_{i} x(k)\right\} \\
:= & \sum_{i=1}^{q} \hat{\Theta}_{i} \mathbb{E}\left\{x^{\mathrm{T}}(k) \Gamma_{i} x(k)\right\}
\end{aligned}
$$

where $\hat{\Theta}_{i}$ and $\Gamma_{i}$ are known matrices with appropriate dimensions, and $q$ is the number of independent state components. 
Remark 1 As pointed out in [21, 26], the nonlinearity description in (2)-(4) covers many well-studied nonlinearities in stochastic systems such as 1) linear system with state- and control-dependent multiplicative noise; 2) nonlinear systems with random vectors dependent on the norms of states and control input; and 3) nonlinear systems with a random sequence dependent on the sign of a nonlinear function of states and control inputs.

It is assumed that the dynamic characteristics of the fault vector $f(k)$ are described as follows:

$$
f(k+1)=A_{f}(k) f(k)
$$

where $A_{f}(k)$ is a known matrix with appropriate dimensions. Note that the fault becomes a constant one when $A_{f}(k) \equiv I$.

The variables $\alpha_{1}(k)$ and $\alpha_{2}(k)$ in (1), which govern the random nature of the occurred faults, are Bernoulli distributed white sequences taking values on 0 or 1 with

$\operatorname{Prob}\left\{\alpha_{1}(k)=1\right\}=\bar{\alpha}_{1}, \operatorname{Prob}\left\{\alpha_{1}(k)=0\right\}=1-\bar{\alpha}_{1}$,

$\operatorname{Prob}\left\{\alpha_{2}(k)=1\right\}=\bar{\alpha}_{2}, \operatorname{Prob}\left\{\alpha_{2}(k)=0\right\}=1-\bar{\alpha}_{2},(6)$

where $\bar{\alpha}_{1} \in[0,1]$ and $\bar{\alpha}_{2} \in[0,1]$ are known constants. It is assumed that $\alpha_{1}(k), \alpha_{2}(k), g(k, x(k))$ and $h(k, x(k))$ are unrelated each other.

Remark 2 The proposed fault modeled by (5) may occur in a probabilistic way based on an individual probability distribution that can be specified a prior through statistical tests. The ROFs concept is to reflect the random fashion of the occurred faults in a networked environment and therefore render more practical significance for the time-varying systems (1). The statistical information of the ROF is used throughout the design of the fault estimators.

We are now ready to state the fault estimation problem under consideration as follows: given a positive scalar $\gamma$, find the fault estimation $\hat{f}(k)(k=0,1, \ldots, N-1)$ such that

$$
\begin{aligned}
& \zeta(N):=\frac{\mathbb{E}\left\{\sum_{k=0}^{N-1}\|\hat{f}(k)-f(k)\|^{2}\right\}}{V_{0}(k)+V_{1}(k)}<\gamma^{2}, \\
& \forall(\{d(k)\},\{v(k)\}, x(0)) \neq 0
\end{aligned}
$$

holds, where $V_{0}(k)=\mathbb{E}\left\{(x(0)-\hat{x}(0))^{T} P_{A}(x(0)-\hat{x}(0))\right\}$, $V_{1}(k)=\sum_{k=0}^{N-1}\left(d^{T}(k) P_{B} d(k)+v^{T}(k) P_{C} v(k)\right), P_{A}, P_{B}$ and $P_{C}$ are known positive definite weighting matrices, and $\hat{x}(0)$ is the estimate of initial state $x(0)$. Without loss of generality, the initial state estimate $\hat{x}(0)$ is assumed to be zero.

Defining $\bar{x}(k)=\left[x^{T}(k) f^{T}(k)\right]^{T}$ and $z(k)=f(k)$, we rewrite (1) and (5) into the following augmented system:

$$
\left\{\begin{aligned}
\bar{x}(k+1)= & \left(\bar{A}(k)+\tilde{\alpha}_{1}(k) \bar{B}_{f}(k)\right) \bar{x}(k)+H g(k, x(k)) \\
& \quad \bar{B}_{d}(k) d(k) \\
y(k)= & \left(\bar{C}(k)+\tilde{\alpha}_{2}(k) \bar{D}_{f}(k)\right) \bar{x}(k)+h(k, x(k)) \\
& +D_{d}(k) v(k) \\
z(k)= & L(k) \bar{x}(k) \\
\bar{x}(0)= & \bar{x}_{0}
\end{aligned}\right.
$$

where

$$
\begin{aligned}
& \bar{A}(k)=\left[\begin{array}{cc}
A(k) & \bar{\alpha}_{1}(k) B_{f}(k) \\
0 & A_{f}(k)
\end{array}\right], \quad \bar{B}_{f}(k)=\left[\begin{array}{cc}
0 & B_{f}(k) \\
0 & 0
\end{array}\right], \\
& \bar{B}_{d}(k)=\left[\begin{array}{lll}
B_{d}^{T}(k) & 0
\end{array}\right]^{T}, \bar{C}(k)=\left[C(k) \bar{\alpha}_{2}(k) D_{f}(k)\right] \text {, } \\
& L(k)=\left[\begin{array}{ll}
0 & I
\end{array}\right], H=\left[\begin{array}{ll}
I & 0
\end{array}\right]^{T}, \quad \bar{D}_{f}(k)=\left[\begin{array}{ll}
0 & D_{f}(k)
\end{array}\right], \\
& \tilde{\alpha}_{1}(k)=\alpha_{1}(k)-\bar{\alpha}_{1}(k), \quad \tilde{\alpha}_{2}(k)=\alpha_{2}(k)-\bar{\alpha}_{2}(k) \text {. }
\end{aligned}
$$

In this paper, the following fault estimator is adopted for the augmented system (8):

$$
\left\{\begin{array}{l}
\hat{\bar{x}}(k+1)=F(k) \hat{\bar{x}}(k)+G(k) y(k) \\
\hat{z}(k)=L(k) \hat{\bar{x}}(k)
\end{array}\right.
$$

where $\hat{\bar{x}}(k) \in \mathbb{R}^{n_{x}+n_{l}}$ is the estimate of the state $\bar{x}(k)$. We assume that $\hat{\bar{x}}(0)=0$. Subsequently, the fault estimation problem can be reformulated as to find $F(k)$ and $G(k)$ such that (7) is satisfied.

Letting $e(k)=\bar{x}(k)-\hat{\bar{x}}(k)$, we have

$$
\begin{aligned}
e(k+1)= & (\bar{A}(k)-F(k)-G(k) \bar{C}(k)) \bar{x}(k)+F(k) e(k) \\
& +\left(\tilde{\alpha}_{1}(k) \bar{B}_{f}(k)-\tilde{\alpha}_{2}(k) G(k) \bar{D}_{f}(k)\right) \bar{x}(k) \\
& +\bar{B}_{d}(k) d(k)-G(k) D_{d}(k) v(k) \\
& +H g(k, x(k))-G(k) h(k, x(k))
\end{aligned}
$$

Denoting $\eta(k):=\left[\begin{array}{ll}\bar{x}^{T}(k) & e^{T}(k)\end{array}\right]^{T}, \tilde{z}(k):=z(k)-\hat{z}(k)$ and $w(k):=\left[\begin{array}{ll}d^{T}(k) & v^{T}(k)\end{array}\right]^{T}$, we have the following system to be investigated:

$$
\left\{\begin{aligned}
& \eta(k+1)=\left(\mathcal{A}(k)+\tilde{\alpha}_{1}(k) \mathcal{B}_{f}(k)+\tilde{\alpha}_{2}(k) \mathcal{D}_{f}(k)\right) \eta(k) \\
&+\mathcal{H}(k) \mathcal{F}(k, x(k))+\mathcal{G}(k) w(k) \\
& \tilde{z}(k)=\mathcal{L}(k) \eta(k)
\end{aligned}\right.
$$

where

$$
\begin{aligned}
& \mathcal{A}(k)=\left[\begin{array}{cc}
\bar{A}(k) & 0 \\
\bar{A}(k)-F(k)-G(k) \bar{C}(k) & F(k)
\end{array}\right], \\
& \mathcal{B}_{f}(k)=\left[\begin{array}{ll}
\bar{B}_{f}(k) & 0 \\
\bar{B}_{f}(k) & 0
\end{array}\right], \quad \mathcal{D}_{f}(k)=\left[\begin{array}{cc}
0 & 0 \\
-G(k) \bar{D}_{f}(k) & 0
\end{array}\right], \\
& \mathcal{H}(k)=\left[\begin{array}{cc}
H & 0 \\
H & -G(k)
\end{array}\right], \quad \mathcal{F}(k, x(k))=\left[\begin{array}{l}
g(k, x(k)) \\
h(k, x(k))
\end{array}\right], \\
& \mathcal{G}(k)=\left[\begin{array}{cc}
\bar{B}_{d}(k) & 0 \\
\bar{B}_{d}(k) & -G(k) D_{d}(k)
\end{array}\right], \quad \mathcal{L}(k)=\left[\begin{array}{ll}
0 & L(k)
\end{array}\right] .
\end{aligned}
$$

Furthermore, the performance requirement (7) is rewritten as

$$
\begin{aligned}
J_{N}:= & \sum_{k=0}^{N-1}\left(\mathbb{E}\left\{\|\tilde{z}(k)\|^{2}\right\}-\gamma^{2} w^{T}(k) P_{\Psi} w(k)\right) \\
& -\gamma^{2} \eta^{T}(0) R \eta(0)<0, \forall(\{w(k)\}, \eta(0)) \neq 0
\end{aligned}
$$


where $R=\operatorname{diag}\left\{0, \bar{P}_{A}\right\}, \bar{P}_{A}=\operatorname{diag}\left\{P_{A}, 0\right\}$ and $P_{\Psi}=$ $\operatorname{diag}\left\{P_{B}, P_{C}\right\}$.

Our objective of this paper is to find the sequence of parameter matrices, $F(k)$ and $G(k)$, such that the dynamic system (11) satisfies the performance requirement (11).

\section{Main results}

Lemma 1 [1] Let matrices $G, M$ and $\Gamma$ be given with appropriate sizes. Then the following matrix equation

$$
G X M=\Gamma
$$

has a solution $X$ if and only if $G G^{\dagger} \Gamma M^{\dagger} M=\Gamma$. Moreover, any solution to (12) is represented by

$$
X=G^{\dagger} \Gamma M^{\dagger}+Y-G^{\dagger} G Y M M^{\dagger}
$$

where $Y$ is a matrix with an appropriate size.

Theorem 1 Consider the time-varying nonlinear stochastic system described by (1)-(5). For a given disturbance attenuation level $\gamma>0$ and the positive definite matrices $P_{B}>0, P_{C}>0$ and $R>0$, the fault estimator $\hat{f}(k)(k=0,1, \ldots, N-1)$ satisfies the performance criterion (7) if and only if the following discrete Riccati difference equation

$$
\begin{aligned}
P(k)= & \mathcal{A}^{T}(k) P(k+1) \mathcal{A}(k)+g_{1}^{2}(k) \mathcal{B}_{f}^{T}(k) P(k+1) \\
& \times \mathcal{B}_{f}(k)+g_{2}^{2}(k) \mathcal{D}_{f}^{T}(k) P(k+1) \mathcal{D}_{f}(k) \\
& +\sum_{i=1}^{q} \hat{\Gamma}_{i} \cdot \operatorname{tr}\left[\mathcal{H}^{T}(k) P(k+1) \mathcal{H}(k) \hat{\Theta}_{i}\right] \\
& +\mathcal{L}^{T}(k) \mathcal{L}(k)+\mathcal{A}^{T}(k) P(k+1) \mathcal{G}(k) \\
& \times \Phi^{-1}(k) \mathcal{G}^{T}(k) P(k+1) \mathcal{A}(k)
\end{aligned}
$$

with

$$
P(N)=0
$$

has a solution $(P(k), F(k), G(k))$ satisfying

$$
\left\{\begin{array}{l}
\Phi(k)=\gamma^{2} P_{\Psi}-\mathcal{G}^{T}(k) P(k+1) \mathcal{G}(k)>0 \\
P(0)<\gamma^{2} R
\end{array}\right.
$$

where $\hat{\Gamma}_{i}=\left[\begin{array}{cc}\bar{\Gamma}_{i} & 0 \\ 0 & 0\end{array}\right], g_{1}(k)=\sqrt{\bar{\alpha}_{1}(k)\left(1-\bar{\alpha}_{1}(k)\right)}, \bar{\Gamma}_{i}=$ $\left[\begin{array}{cc}\Gamma_{i} & 0 \\ 0 & 0\end{array}\right], g_{2}(k)=\sqrt{\bar{\alpha}_{2}(k)\left(1-\bar{\alpha}_{2}(k)\right)}$ and $P_{\Psi}$ is defined in (11).

Proof: Sufficiency. Define $J(k)=\eta^{T}(k+1) P(k+$ 1) $\eta(k+1)-\eta^{T}(k) P(k) \eta(k)$. Noticing (11) and taking mathematical expectation as follows, we have

$$
\begin{aligned}
& \mathbb{E}\{J(k)\} \\
= & \mathbb{E}\left\{\left(\left(\mathcal{A}(k)+\tilde{\alpha}_{1}(k) \mathcal{B}_{f}(k)+\tilde{\alpha}_{2}(k) \mathcal{D}_{f}(k)\right) \eta(k)+\mathcal{H}(k)\right.\right. \\
& \times \mathcal{F}(k, x(k))+\mathcal{G}(k) w(k))^{T} P(k+1)\left(\left(\mathcal{A}(k)+\tilde{\alpha}_{1}(k)\right.\right. \\
& \left.\times \mathcal{B}_{f}(k)+\tilde{\alpha}_{2}(k) \mathcal{D}_{f}(k)\right) \eta(k)+\mathcal{H}(k) \mathcal{F}(k, x(k)) \\
& \left.+\mathcal{G}(k) w(k))-\eta^{T}(k) P(k) \eta(k)\right\}
\end{aligned}
$$

Taking (4) into consideration, we have

$$
\begin{aligned}
& \mathbb{E}\left\{\mathcal{F}^{T}(k, x(k)) \mathcal{H}^{T}(k) P(k+1) \mathcal{H}(k) \mathcal{F}(k, x(k))\right\} \\
= & \mathbb{E}\left\{\eta^{T}(k) \sum_{i=1}^{q} \hat{\Gamma}_{i} \cdot \operatorname{tr}\left[\mathcal{H}^{T}(k) P(k+1) \mathcal{H}(k) \hat{\Theta}_{i}\right] \eta(k)\right\} .
\end{aligned}
$$

Adding the following zero term $\|\tilde{z}(k)\|^{2}-\gamma^{2} w^{T}(k) P_{\Psi} w(k)-\|\tilde{z}(k)\|^{2}+\gamma^{2} w^{T}(k) P_{\Psi} w(k)$

to the right side of (15) results in

$$
\begin{aligned}
& \mathbb{E}\{J(k)\} \\
= & \mathbb{E}\left\{\eta ^ { T } ( k ) \left(\mathcal{A}^{T}(k) P(k+1) \mathcal{A}(k)+g_{1}^{2}(k) \mathcal{B}_{f}^{T}(k) P(k+1)\right.\right. \\
& \times \mathcal{B}_{f}(k)+g_{2}^{2}(k) \mathcal{D}_{f}^{T}(k) P(k+1) \mathcal{D}_{f}(k)+\mathcal{L}^{T}(k) \mathcal{L}(k) \\
& \left.+\sum_{i=1}^{q} \hat{\Gamma}_{i} \cdot \operatorname{tr}\left[\mathcal{H}^{T}(k) P(k+1) \mathcal{H}(k) \hat{\Theta}_{i}\right]-P(k)\right) \eta(k) \\
& +2 \eta^{T}(k) \mathcal{A}^{T}(k) P(k+1) \mathcal{G}(k) w(k)-w^{T}(k) \Phi(k) w(k) \\
& \left.-\left(\|\tilde{z}(k)\|^{2}-\gamma^{2} w^{T}(k) P_{\Psi} w(k)\right)\right\} .
\end{aligned}
$$

By the completing squares method, it is not difficult to see that

$$
\begin{aligned}
& \mathbb{E}\{J(k)\} \\
= & \mathbb{E}\left\{\eta ^ { T } ( k ) \left(\mathcal{A}^{T}(k) P(k+1) \mathcal{A}(k)+g_{1}^{2}(k) \mathcal{B}_{f}^{T}(k) P(k+1)\right.\right. \\
& \times \mathcal{B}_{f}(k)+g_{2}^{2}(k) \mathcal{D}_{f}^{T}(k) P(k+1) \mathcal{D}_{f}(k)+\mathcal{L}^{T}(k) \mathcal{L}(k) \\
& +\sum_{i=1}^{q} \hat{\Gamma}_{i} \cdot \operatorname{tr}\left[\mathcal{H}^{T}(k) P(k+1) \mathcal{H}(k) \hat{\Theta}_{i}\right]+\mathcal{A}^{T}(k) \\
& \left.\times P(k+1) \mathcal{G}(k) \Phi^{-1}(k) \mathcal{G}^{T}(k) P(k+1) \mathcal{A}(k)\right) \eta(k) \\
& -P(k)\}-\mathbb{E}\left\{\left(w(k)-w^{*}(k)\right)^{T} \Phi(k)\left(w(k)-w^{*}(k)\right)\right\} \\
& -\mathbb{E}\left\{\|\tilde{z}(k)\|^{2}-\gamma^{2} w^{T}(k) P_{\Psi} w(k)\right\}
\end{aligned}
$$

where

$$
w^{*}(k)=\Phi^{-1}(k) \mathcal{G}^{T}(k) P(k+1) \mathcal{A}(k) \eta(k) .
$$

Taking the sum on both sides of (18) from 0 to $N-1$, we obtain

$$
\begin{aligned}
& \mathbb{E}\left\{\sum_{k=0}^{N-1} J(k)\right\} \\
= & -\sum_{k=0}^{N-1} \mathbb{E}\left\{\left(w(k)-w^{*}(k)\right)^{T} \Phi(k)\left(w(k)-w^{*}(k)\right)\right\} \\
& -\sum_{k=0}^{N-1} \mathbb{E}\left\{\|\tilde{z}(k)\|^{2}-\gamma^{2} w^{T}(k) P_{\Psi} w(k)\right\}
\end{aligned}
$$


By noticing $\Phi(k)>0, P(0)-\gamma^{2} R<0$, the final condition $P(N)=0$ and $\eta(0) \neq 0$, we obtain

$$
\begin{aligned}
J_{N}:= & \mathbb{E}\left\{\sum_{k=0}^{N-1}\left(\|\tilde{z}(k)\|^{2}-\gamma^{2} w^{T}(k) P_{\Psi} w(k)\right)\right\} \\
& -\gamma^{2} \mathbb{E}\left\{\eta^{T}(0) R \eta(0)\right\} \\
= & \mathbb{E}\left\{\eta^{T}(0)\left(P(0)-\gamma^{2} R\right) \eta(0)\right\}-\sum_{k=0}^{N-1} \mathbb{E}\{(w(k) \\
& \left.\left.-w^{*}(k)\right)^{T} \Phi(k)\left(w(k)-w^{*}(k)\right)\right\}<0
\end{aligned}
$$

which is equivalent to (11). Hence we finish completing the proof of sufficiency.

Necessity: The proof follows directly from that of Theorem 1 in [24] and is therefore omitted.

Remark 3 So far, a necessary and sufficient condition has been obtained in Theorem 1 for the existence of the fault estimation performance of the dynamic system (11). That is, if there exists a solution $P(k)$ to (13) such that $\Phi(k)>0$ and $P(0)<\gamma^{2} R$, then the fault estimation $\hat{f}(k)(k=0,1, \ldots, N-1)$ satisfies the performance criterion (7). Moreover, according to (21), the worst-case disturbance can be expressed as $w^{*}(k)=\Phi^{-1}(k) \mathcal{G}^{T}(k) P(k+1) \mathcal{A}(k) \eta(k)$, and the performance objective $J_{N}=\eta^{T}(0)\left(P(0)-\gamma^{2} R\right) \eta(0)$. In the main results, the occurring probability of the random faults is reflected. For probability 1, our results reduce to those for the traditional deterministic fault estimation problem and, in the case of probability 0 , the fault estimation problem is no longer valid and our developed approach applies to the state estimation problem only. In the case that all the time-varying parameters become time-invariant (constant), (13)-(14) would become a rather standard Riccati difference equation whose feasibility has been widely investigated in the literature, see e.g. [32].

In what follows, we aim to determine the gain matrices $F(k)$ and $G(k)$ of the desired fault estimator under the situation of the worst-case disturbance $w^{*}(k)$.

Theorem 2 Consider the time-varying nonlinear stochastic system described by (1)-(5). Let a disturbance attenuation level $\gamma>0$ and the positive definite matrices $P_{B}>0, P_{C}>0$ and $R>0$ be given. For each $k=0,1, \ldots, N-1$, assume that the discrete Riccati difference equation (13) has a solution $(P(k), F(k), G(k))$ satisfying (14) and the following discrete Riccati difference equation:

$$
\begin{aligned}
Q(k)= & \mathcal{A}_{G}^{T}(k) Q(k+1) \mathcal{A}_{G}(k)+g_{1}^{2}(k) \mathcal{B}_{f}^{T}(k) Q(k+1) \\
& \times \mathcal{B}_{f}(k)+g_{2}^{2}(k) \mathcal{D}_{f}^{T}(k) Q(k+1) \mathcal{D}_{f}(k) \\
& +\sum_{i=1}^{q} \hat{\Gamma}_{i} \cdot \operatorname{tr}\left[\mathcal{H}^{T}(k) Q(k+1) \mathcal{H}(k) \hat{\Theta}_{i}\right] \\
& +\mathcal{L}^{T}(k) \mathcal{L}(k)-\mathcal{A}_{G}^{T}(k) Q(k+1) \Omega^{-1}(k) \\
& \times Q(k+1) \mathcal{A}_{G}(k)
\end{aligned}
$$

with

$$
Q(N)=0, \quad \mathcal{N}(k)=\mathcal{N}(k) \mathcal{C}^{\dagger}(k) \mathcal{C}(k)
$$

has a solution $(Q(k), \mathcal{K}(k))$ satisfying

$\Omega(k)=Q(k+1)+I>0$

where

$$
\begin{aligned}
\mathcal{N}(k) & =-\bar{L} \Omega^{-1}(k) Q(k+1) \mathcal{A}_{G}(k), \\
\mathcal{C}(k) & =\left[\begin{array}{cc}
-I & I \\
-\bar{C}(k) & 0
\end{array}\right], \overline{\mathcal{A}}(k)=\left[\begin{array}{cc}
\bar{A}(k) & 0 \\
\bar{A}(k) & 0
\end{array}\right], \\
\mathcal{A}_{G}(k) & =\overline{\mathcal{A}}(k)+\mathcal{G}(k) \Phi^{-1}(k) \mathcal{G}^{T}(k) P(k+1) \mathcal{A}(k), \\
\mathcal{K}(k) & =[F(k) G(k)] .
\end{aligned}
$$

Then, we can conclude that the fault estimation $\hat{f}(k)$ $(k=0,1, \ldots, N-1)$ satisfies the performance criterion (7) and the gain matrices of the fault estimator are given by

$$
\begin{aligned}
& \mathcal{K}(k)=[F(k) G(k)] \\
= & \mathcal{N}(k) \mathcal{C}^{\dagger}(k)+Y(k)-Y(k) \mathcal{C}(k) \mathcal{C}^{\dagger}(k), \\
& Y(k) \in \mathbb{R}^{n_{x}+n_{l} \times\left(n_{x}+n_{l}+n_{y}\right)}, k=1,2, \ldots, N-1 .
\end{aligned}
$$

Proof: We define a cost function as follows:

$J_{N}\left(w^{*}\right)=\mathbb{E}\left\{\sum_{k=0}^{N-1}\left(\|\tilde{z}(k)\|^{2}+\|\Upsilon(k)\|^{2}\right)\right\}$

where $\Upsilon(k)=\mathcal{K}(k) \mathcal{C}(k) \eta(k)$. Therefore, the original system (11) with the worst-case disturbance $w^{*}(k)$ can be rewritten as follows:

$$
\left\{\begin{aligned}
\eta_{k+1}= & \left(\mathcal{A}_{G}(k)+\tilde{\alpha}_{1}(k) \mathcal{B}_{f}(k)+\tilde{\alpha}_{2}(k) \mathcal{D}_{f}(k)\right) \eta(k) \\
& +\mathcal{H}(k) \mathcal{F}(k, x(k))+\tilde{\Upsilon}(k) \\
\tilde{z}(k)= & \mathcal{L}(k) \eta(k)
\end{aligned}\right.
$$

where $\tilde{\Upsilon}(k)=\left[\begin{array}{ll}0 & \Upsilon(k)^{T}\end{array}\right]^{T}$.

In order to obtain the parametric expression of $\mathcal{K}(k)$, we define

$$
J_{w}(k)=\eta^{T}(k+1) Q(k+1) \eta(k+1)-\eta^{T}(k) Q(k) \eta(k) .
$$

It follows from (27) that

$$
\begin{aligned}
& \mathbb{E}\left\{J_{w}(k)\right\} \\
= & \mathbb{E}\left\{\left(\left(\mathcal{A}_{G}(k)+\tilde{\alpha}_{1}(k) \mathcal{B}_{f}(k)+\tilde{\alpha}_{2}(k) \mathcal{D}_{f}(k)\right) \eta(k)\right.\right. \\
& +\mathcal{H}(k) \mathcal{F}(k, x(k))+\tilde{\Upsilon}(k))^{T} Q(k+1)\left(\left(\mathcal{A}_{G}(k)\right.\right. \\
& \left.+\tilde{\alpha}_{1}(k) \mathcal{B}_{f}(k)+\tilde{\alpha}_{2}(k) \mathcal{D}_{f}(k)\right) \eta(k)+\mathcal{H}(k) \mathcal{F}(k, x(k)) \\
& \left.+\tilde{\Upsilon}(k))-\eta^{T}(k) Q(k) \eta(k)\right\}+\mathbb{E}\left\{\|\tilde{z}(k)\|^{2}+\|\Upsilon(k)\|^{2}\right. \\
& \left.-\|\tilde{z}(k)\|^{2}-\|\Upsilon(k)\|^{2}\right\} .
\end{aligned}
$$


By applying completing squares method again, we have

$$
\begin{aligned}
& \mathbb{E}\left\{J_{w}(k)\right\} \\
= & \mathbb{E}\left\{\eta ^ { T } ( k ) \left(\mathcal{A}_{G}^{T}(k) Q(k+1) \mathcal{A}_{G}(k)+g_{1}^{2}(k) \mathcal{B}_{f}^{T}(k)\right.\right. \\
& \times Q(k+1) \mathcal{B}_{f}(k)+g_{2}^{2}(k) \mathcal{D}_{f}^{T}(k) Q(k+1) \mathcal{D}_{f}(k) \\
& +\sum_{i=1}^{q} \hat{\Gamma}_{i} \cdot \operatorname{tr}\left[\mathcal{H}^{T}(k) Q(k+1) \mathcal{H}(k) \hat{\Theta}_{i}\right]+\mathcal{L}^{T}(k) \mathcal{L}(k) \\
& \left.-Q(k)-\mathcal{A}_{G}^{T}(k) Q(k+1) \Omega^{-1}(k) Q(k+1) \mathcal{A}_{G}(k)\right) \\
& \left.\times \eta(k)+\left(\tilde{\Upsilon}(k)-\tilde{\Upsilon}^{*}(k)\right)^{T} \Omega(k)\left(\tilde{\Upsilon}(k)-\tilde{\Upsilon}^{*}(k)\right)\right\} \\
& -\mathbb{E}\left\{\|\tilde{z}(k)\|^{2}+\|\Upsilon(k)\|^{2}\right\}
\end{aligned}
$$

where $\tilde{\Upsilon}^{*}(k):=-\Omega^{-1}(k) Q(k+1) \mathcal{A}_{G}(k) \eta(k)$. Therefore, it is true that

$$
\begin{aligned}
& J_{N}\left(w^{*}\right) \\
= & \mathbb{E}\left\{\sum_{k=0}^{N-1}\left(\|\tilde{z}(k)\|^{2}+\|\Upsilon(k)\|^{2}\right)\right\} \\
= & \mathbb{E}\left\{\sum_{k=0}^{N-1}\left(\tilde{\Upsilon}(k)-\tilde{\Upsilon}^{*}(k)\right)^{T} \Omega(k)\left(\tilde{\Upsilon}(k)-\tilde{\Upsilon}^{*}(k)\right)\right. \\
& +\eta^{T}(0) Q(0) \eta(0)-\eta^{T}(N) Q(N) \eta(N)+\sum_{k=0}^{N-1} \eta^{T}(k) \\
& \times\left(\mathcal{A}_{G}^{T}(k) Q(k+1) \mathcal{A}_{G}(k)+g_{1}^{2}(k) \mathcal{B}_{f}^{T}(k) Q(k+1) \mathcal{B}_{f}(k)\right. \\
& +g_{2}^{2}(k) \mathcal{D}_{f}^{T}(k) Q(k+1) \mathcal{D}_{f}(k)+\mathcal{L}^{T}(k) \mathcal{L}(k)-Q(k) \\
& +\sum_{i=1}^{q} \hat{\Gamma}_{i} \cdot \operatorname{tr}\left[\mathcal{H}^{T}(k) Q(k+1) \mathcal{H}(k) \hat{\Theta}_{i}\right]-\mathcal{A}_{G}^{T}(k) Q(k+1) \\
& \left.\left.\times \Omega^{-1}(k) Q(k+1) \mathcal{A}_{G}(k)\right) \eta(k)\right\}
\end{aligned}
$$

Under the zero final condition of $Q(N)$, in order to minimize the cost of $J_{N}\left(w^{*}\right)$, the best choice of $\mathcal{K}(k)$ is to satisfy the following condition:

$$
\left\{\begin{aligned}
& Q(k)= \mathcal{A}_{G}^{T}(k) Q(k+1) \mathcal{A}_{G}(k)+g_{1}^{2}(k) \mathcal{B}_{f}^{T}(k) Q(k+1) \\
& \times \mathcal{B}_{f}(k)+g_{2}^{2}(k) \mathcal{D}_{f}^{T}(k) Q(k+1) \mathcal{D}_{f}(k)+\mathcal{L}^{T}(k) \\
& \times \mathcal{L}(k)+\sum_{i=1}^{q} \hat{\Gamma}_{i} \cdot \operatorname{tr}\left[\mathcal{H}^{T}(k) Q(k+1) \mathcal{H}(k) \hat{\Theta}_{i}\right] \\
&-\mathcal{A}_{G}^{T}(k) Q(k+1) \Omega^{-1}(k) Q(k+1) \mathcal{A}_{G}(k) \\
& \mathcal{K}(k) \mathcal{C}(k)=-\bar{L} \Omega^{-1}(k) Q(k+1) \mathcal{A}_{G}(k)
\end{aligned}\right.
$$

where $\bar{L}=\left[\begin{array}{ll}0 & I\end{array}\right]$. According to Lemma 1 , it can be observed that the existence of a solution $\mathcal{K}(k)(k=$
$0,1, \ldots, N-1)$ is equivalent to the feasibility of

$$
\begin{aligned}
& -\bar{L} \Omega^{-1}(k) Q(k+1) \mathcal{A}_{G}(k) \mathcal{C}^{\dagger}(k) \mathcal{C}(k) \\
= & -\bar{L} \Omega^{-1}(k) Q(k+1) \mathcal{A}_{G}(k)
\end{aligned}
$$

whose general solution is given by

$$
\begin{aligned}
K_{k}= & -\bar{L} \Omega^{-1}(k) Q(k+1) \mathcal{A}_{G}(k) \mathcal{C}^{\dagger}(k)+Y(k) \\
& -Y(k) \mathcal{C}(k) \mathcal{C}^{\dagger}(k)
\end{aligned}
$$

where $Y(k)$ is any matrix with dimension $n_{x}+n_{l} \times\left(n_{x}\right.$ $\left.+n_{l}+n_{y}\right)$. The proof of this theorem is now complete.

By means of Theorem 2, we can summarize the FiniteHorizon Fault Estimator Design (FHFED) algorithm as follows:

\section{Algorithm FHFED}

Step 1: Given the disturbance attenuation level $\gamma$, the positive definite matrices $P_{B}>0, P_{C}>0$ and $R>0$, set $k=N-1$.

Step 2: Calculate $\Phi(k), \Omega(k)$ and $\mathcal{N}(k)$ with known $P(k+1)$ and $Q(k+1)$ via the first equation of (14) and equations (23) and (24), respectively. Furthermore, the fault estimation gain matrix $\mathcal{K}(k)$ can be obtained by equation (25).

Step 3: If $\mathcal{N}(k)=\mathcal{N}(k) \mathcal{C}^{\dagger}(k) \mathcal{C}(k)$, then solve the first equation of (13) and (22) to get $P(k)$ and $Q(k)$, respectively, and go to the next step, else this algorithm is infeasible, stop.

Step 4: If $k \neq 0, \Phi(k)>0$ and $\Omega(k)>0$, set $k=k-1$ and go to Step 2, else go to the next step.

Step 5: If $P(0) \geq r^{2} R$ or $\Phi(k) \leq 0$ or $\Omega(k) \leq 0$, then this algorithm is infeasible, stop.

Remark 4 In this paper, a coupled Riccati difference equation (RDE) approach is proposed to solve the estimation problem of the randomly occurring faults over a finite-horizon. The faults are allowed to occur dynamically governed by random variables with given probability laws. It can be observed from Algorithm FHFED that, in the estimator design procedure, all the important factors contributing to the system complexity have been reflected which include 1) the time-varying systems parameters; 2) the occurrence probabilities of the random faults; 3) statistics information about the stochastic nonlinearities; and 4) the prescribed disturbance attenuation level. The coupled RDE algorithm is backward recursive and therefore suitable for online application. The main theoretical contribution would be the establishment of the necessary and sufficient conditions for the existence of the desired finite-horizon $H_{\infty}$ fault estimator.

\section{An Illustrative Example}

In this section, we present a simulation example to illustrate the effectiveness of the proposed finite-horizon 
estimation of randomly occurring faults for a class of nonlinear time-varying systems.

Consider a discrete time-varying system described by (1)-(5) with the following parameters over the finite time-horizon $[0,50]$ :

$$
\begin{aligned}
& A(k)=\left[\begin{array}{cc}
0 & -0.7 \\
0.1+0.2 \sin (3 k) & -0.53
\end{array}\right], B_{f}(k)=\left[\begin{array}{l}
0.4 \\
0.2
\end{array}\right], \\
& C(k)=\left[\begin{array}{cc}
-0.2+0.1 \sin (5 k) & 0.5 \\
0.1 & 1
\end{array}\right], D_{d}(k)=\left[\begin{array}{ll}
0.2 & 0.5
\end{array}\right]^{T}, \\
& D_{f}(k)=\left[\begin{array}{ll}
1 & 1
\end{array}\right]^{T}, B_{d}(k)=\left[\begin{array}{ll}
0.2 & 0.5
\end{array}\right]^{T}, L(k)=\left[\begin{array}{ll}
0 & I
\end{array}\right], \\
& g(k, x(k))=\left[\begin{array}{l}
0.1 \\
0.3
\end{array}\right] \times\left(0.2 x_{1}(k) \xi_{1}(k)+0.3 x_{2}(k) \xi_{2}(k)\right), \\
& h(k, x(k))=\left[\begin{array}{l}
0.1 \\
0.1
\end{array}\right] \times\left(0.2 x_{1}(k) \xi_{1}(k)+0.3 x_{2}(k) \xi_{2}(k)\right),
\end{aligned}
$$

where $x_{i}(k)(i=1,2)$ is the $i$ th element of $x(k)$, and $\xi_{i}(k)(i=1,2)$ are zero mean, uncorrelated Gaussian white noise processes with unity variances that is also uncorrelated with $d(k)$ and $v(k)$. It can be easily checked that the above class of stochastic nonlinearities satisfies

$$
\begin{aligned}
& \mathbb{E}\left\{\left[\begin{array}{l}
g(k, x(k)) \\
h(k, x(k))
\end{array}\right] \mid x(k)\right\}=0 \\
& \mathbb{E}\left\{\left[\begin{array}{l}
g(k, x(k)) \\
h(k, x(k))
\end{array}\right]\left[g^{T}(k, x(k)) h^{T}(k, x(k))\right] \mid x(k)\right\} \\
& =\left[\begin{array}{l}
0.1 \\
0.3 \\
0.1 \\
0.1
\end{array}\right]\left[\begin{array}{l}
0.1 \\
0.3 \\
0.1 \\
0.1
\end{array}\right] \mathbb{E}\left\{x^{T}(k)\left[\begin{array}{cc}
0.04 & 0 \\
0 & 0.09
\end{array}\right] x(k)\right\} .
\end{aligned}
$$

The $H_{\infty}$ performance level $\gamma$, the positive definite matrices $P_{B}, P_{C}$ and $R$ are chosen as $\gamma=1, P_{B}=I, P_{C}=I$ and $R=\operatorname{diag}\{0,0,0,2,2,0\}$, respectively. Let $\bar{\alpha}_{1}=0.9$ and $\bar{\alpha}_{2}=0.8$. Using the developed computational algorithm and Matlab (with the YALMIP 3.0), we can check the feasibility of the coupled recursive RDEs and obtain the desired fault estimate parameters which are listed in table 1 from the time $k=0$ to $k=4$. In the simulation, the initial values of the states are $\bar{x}(0)=\left[\begin{array}{lll}0.2 & -0.6 & 0\end{array}\right]^{T}$ and $\hat{\bar{x}}(0)=\left[\begin{array}{lll}0 & 0 & 0\end{array}\right]^{T}$, the exogenous disturbance input is selected as $d(k)=0.2 \cos (k)$ and the measure noise is $v(k)=0.3 \sin (k)$.

First, let the matrix $A_{f}(k)=I$. We assume that the fault to be estimated is $f(k)=2$. Fig. 1 plots the simulation result on the fault signal and its estimate. Fig. 2 shows the evolution of the actual fault estimation performance in terms of the index $\zeta(N)$ in (7), from which it can be

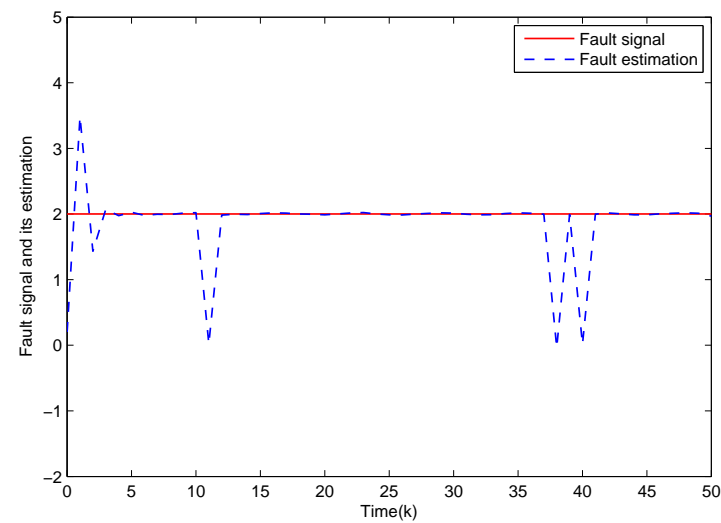

Fig. 1. Fault signal and its estimate

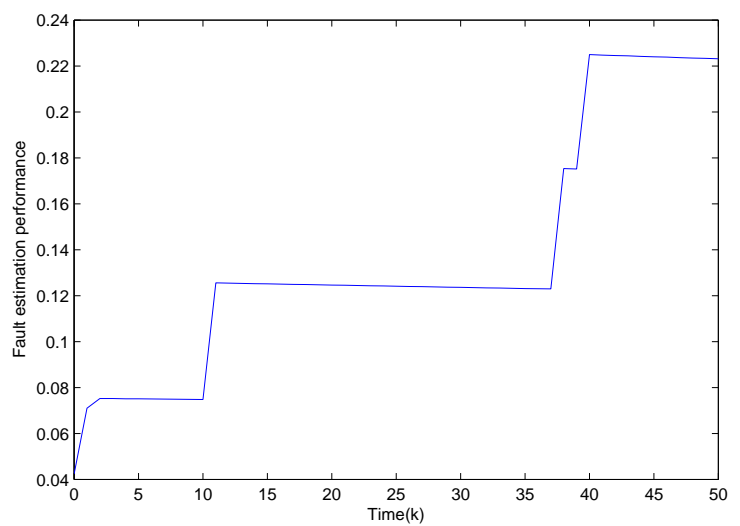

Fig. 2. Fault estimation performance $(\zeta(k))$

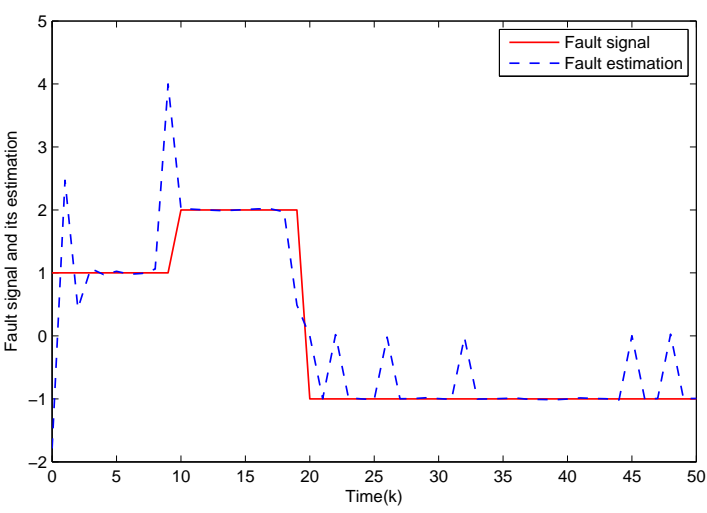

Fig. 3. Fault signal and its estimate

seen that the index $\zeta(N)(N=1,2, \ldots, 50)$ is always less than the prescribed upper bound 1 . Next, in order to examine the effects of the fault estimation over different 
Table 1

Fault estimate parameters

\begin{tabular}{c|lccc}
\hline$k$ & 1 & 2 & 3 \\
\hline \multirow{3}{*}{$F(k)$} & {$\left[\begin{array}{ccc}0.3331 & 0 & 0 \\
0 & 0.2220 & 0 \\
0 & 0 & 0\end{array}\right]$} & {$\left[\begin{array}{ccc}0.011 & 0 & 0 \\
0 & 0.2220 & 0.02 \\
0.509 & 0.001 & 0\end{array}\right]$} & {$\left[\begin{array}{ccc}0.0061 & 0.0032 & 0.0011 \\
0.0032 & 0.0107 & 0 \\
0.0011 & 0 & 0.021\end{array}\right]$} \\
\hline$G(k)$ & {$\left[\begin{array}{cc}0.0000 & 0.0000 \\
0.0000 & 0.0000 \\
20000 & -1.0000\end{array}\right]$} & {$\left[\begin{array}{ccc}0.0155 & -0.0001 \\
-0.0001 & 0.0153 \\
-0.0046 & 0.0023\end{array}\right]$} & {$\left[\begin{array}{ccc}0.0037 & -0.0002 \\
-0.0000 & 0.0031 \\
-1.0100 & 2.0252\end{array}\right]$} \\
\hline
\end{tabular}

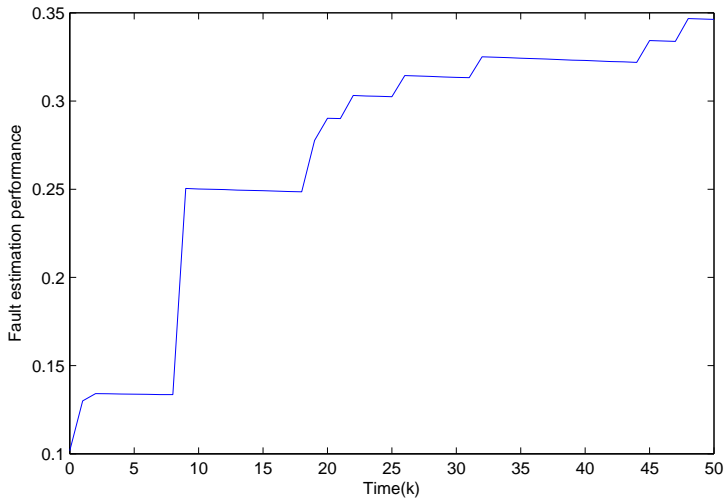

Fig. 4. Fault estimation performance $(\zeta(k))$

time-intervals, we choose the matrix $A_{f}(k)$ as follows:

$$
A_{f}(k)=\left\{\begin{array}{cc}
I & 0 \leq k \leq 9 \\
2 I & k=10 \\
I & 11 \leq k \leq 19 \\
-0.5 I & k=20 \\
I & \text { else }
\end{array}\right.
$$

The fault to be estimated is $f(k)=1$. Fig. 3 plots the fault signal and its estimate. The actual fault estimation performance is depicted in Fig. 4. It can be seen that the developed approach provides the desired performance for the addressed fault estimation problem.

\section{Conclusion}

In this paper, we have dealt with the finite-horizon fault estimation problem for a class of nonlinear stochastic time-varying systems with randomly occurring faults. All the system parameters are time-varying and the stochastic nonlinearities under consideration could cover several classes of well-studied nonlinearities. The failures occur in a random way, and two sets of Bernoulli distributed white sequences have been introduced to govern the fault occurrence probability. The solvability of the addressed fault estimation problem has been dealt with by using the completing squares method and stochastic analysis techniques. The time-varying parameters of the fault estimator have been obtained by solving coupled backward recursive Riccati difference equations. An illustrative example has been used to highlight the effectiveness of the proposed fault estimation technology presented in this paper. It should be noted that one of the future research topics would be to investigate the problems of fault estimation and faulttolerant control for nonlinear polynomial systems $[8,9]$ over a finite-horizon.

\section{References}

[1] M. Aitrami, X. Chen and X. Zhou, Discrete-time indefinite LQ control with state and control dependent noises, Journal of Global Optimization, Vol. 23, No. 3-4, pp. 245-265, 2002.

[2] M. V. Basin and M. A. Pinsky, Stability impulse control of faulted nonlinear systems, IEEE Trans. Automatic Control, Vol. 43, No. 11, pp. 1604-1608, 1998.

[3] S. X. Ding, T. Jeinsch, P. M. Frank and E. L. Ding, A unified approach to the optimization of fault detection systems, Int. J. Adaptive Control and Signal Processing, Vol. 14, No. 7, pp. $725-745,2000$.

[4] H. Dong, Z. Wang and H. Gao, Filtering, Control and Fault Detection with Randomly Occurring Incomplete Information, Wiley, Chichester, UK, 2013.

[5] H. Dong, Z. Wang and H. Gao, Distributed $H_{\infty}$ filtering for a class of Markovian jump nonlinear time-delay systems over lossy sensor networks, IEEE Trans. Industrial Electronics, Vol. 60, No. 10, pp. 4665-4672, 2013.

[6] Z. Gao and S. X. Ding, Actuator fault robust estimation and fault-tolerant control for a class of nonlinear descriptor systems, Automatica, Vol. 43, No. 5, pp. 912-920, 2007.

[7] X. He, Z. Wang and D. Zhou, Robust fault detection for networked systems with communication delay and data missing, Automatica, Vol. 45, No. 11, pp. 2634-2639, 2009.

[8] M. Hernandez-Gonzalez and M. V. Basin, Discrete-time optimal control for stochastic nonlinear polynomial systems, Int. J. General Systems, Vol. 43, No. 3-4, pp. 359-371, 2014.

[9] M. Hernandez-Gonzalez and M. V. Basin, Discrete-time filtering for nonlinear polynomial systems over linear observations, Int. J. Systems Science, Vol. 45, No. 7, pp. 1461-1472, 2014.

[10] J. Hu, Z. Wang, H. Gao and L.K. Stergioulas, Robust sliding mode control for discrete stochastic systems with mixed time delays, randomly occurring uncertainties, and randomly occurring nonlinearities, IEEE Trans. Ind. Electron., Vol. 59, No. 7, pp. 3008-3016, 2012.

[11] B. Jiang, K. Zhang and P. Shi, Integrated fault estimation and accommodation design for discrete-time Takagi-Sugeno fuzzy systems with actuator faults, IEEE Trans. Fuzzy Systems, Vol. 20, No. 1, pp. 192-200, 2011.

[12] B. Jiang, M. Staroswiecki and V. Cocquempot, Fault accommodation for nonlinear dynamic systems, IEEE Trans. Automatic Control, Vol. 51, No. 9, pp. 1578-1583, 2006.

[13] B. Jiang, P. Shi and Z. Mao, Sliding mode observer-based fault estimation for nonlinear networked control systems, Circuits Syst Signal Process Vol. 30, pp. 1-16, 2011. 
[14] H. R. Karimi, Robust synchronization and fault detection of uncertain master-slave systems with mixed time-varying delays and nonlinear perturbations, Int. J. Control, Automation, and Systems, Vol. 9, No. 4, pp. 671-680, 2011.

[15] B. Kulcsar and M. Verhaegen, Robust inversion based fault estimation for discrete-time LPV systems, IEEE Trans. Automatic Control, Vol. 57, No. 6, pp. 1581-1586, 2012.

[16] C. Keliris, M. M. Polycarpou and T. Parisini, A distributed fault detection filtering approach for a class of interconnected continuous-time nonlinear systems, IEEE Trans. Automatic Control, Vol. 58, No. 8, pp. 2032-2047, 2013.

[17] B. Shen, S. X. Ding and Z. Wang, Finite-horizon fault estimation for linear discrete time-varying systems with delayed measurements, Automatica, Vol. 49, No. 1, pp. 293296, 2013.

[18] B. Shen, Z. Wang, H. Shu and G. Wei, $H_{\infty}$ filtering for uncertain time-varying systems with multiple randomly occurred nonlinearities and successive packet dropouts, International Journal of Robust and Nonlinear Control, Vol. 21, No. 14, pp. 1693-1709, 2011.

[19] D. Xu, B. Jiang and P. Shi, Nonlinear actuator fault estimation observer: An inverse system approach via a T-S fuzzy model, Applied Mathematics and Computer Science, Vol. 22, No. 1, pp. 183-196, 2012.

[20] P. Zhang, S. X. Ding and P. Liu, A lifting based approach to observer based fault detection of linear periodic systems, IEEE Trans. Automatic Control, Vol. 57, No. 2, pp. 457-462, 2012.

[21] W. NaNacara and E. E. Yaz, Recursive estimator for linear and nonlinear systems with uncertain observations, Signal Processing, Vol. 62, No. 2, pp. 215-228, 1997.

[22] Y. Wang, S. X. Ding, H. Ye and G. Wang, A new fault detection scheme for networked control systems subject to uncertain time-varying delay, IEEE Trans. Signal Processing, Vol. 56, No. 10, pp. 2558-2568, 2008.

[23] Z. Wang, B. Shen and X. Liu, $H_{\infty}$ filtering with randomly occurring sensor saturations and missing measurements, Automatica, Vol. 48, No. 3, pp. 556-562, 2012.

[24] Z. Wang, H. Dong, B. Shen and H. Gao, Finite-horizon $H_{\infty}$ filtering with missing measurements and quantization effects, IEEE Trans. Automatic Control, Vol. 58, No. 7, pp. 17071718, 2013.

[25] L. Wu, X. Yao and W. X. Zheng, Generalized $H_{2}$ fault detection for two-dimensional Markovian jump systems, Automatica, Vol. 48, No. 8, pp. 1741-1750, 2013.

[26] Y. I. Yaz and E. E. Yaz, On LMI formulations of some problems arising in nonlinear stochastic system analysis, IEEE Trans. Automatic Control, Vol. 44, No. 4, pp. 813-816, 1999.

[27] X. Yan and C. Edwards, Nonlinear robust fault reconstruction and estimation using a sliding mode observer, Automatica Vol. 43, No. 9, pp. 1605-1614, 2007.

[28] X. Yao and L. Guo, Composite anti-disturbance control for Markovian jump nonlinear systems via disturbance observer, Automatica, Vol. 49, No. 8, pp. 2538-2545, 2013.

[29] K. Zhang, B. Jiang and V. Cocquempot, Adaptive observerbased fast fault estimation, Int. J. Control, Automation, and Systems, Vol. 6, No. 3, pp. 320-326, 2008.

[30] M. Zhong, S. X. Ding, Q. Han and Q. Ding, Parity space-based fault estimation for linear discrete time-varying systems, IEEE Trans. Automatic Control, Vol. 55, No. 7, pp. 1726-1731, 2010.

[31] M. Zhong, D. Zhou and S. X. Ding, On designing $H_{\infty}$ fault detection filter for linear discrete time-varying systems, IEEE Trans. Automatic Control, Vol. 55, No. 7, pp. 1689-1695, 2010.

[32] P. Lancaster and L. Rodman, Algebraic Riccati Equations, Series: Oxford Science Publications, Oxford University Press, 1995.
Hongli Dong received the Ph.D. degree in Control Science and Engineering in 2012 from Harbin Institute of Technology, Harbin, China. From July 2009 to January 2010, she was a Research Assistant in the Department of Applied Mathematics, the City University of Hong Kong. From October 2010 to January 2011, she was a Research Assistant in the Department of Mechanical Engineering, the University of Hong Kong. From January 2011 to January 2012, she was a Visiting Scholar in the Department of Information Systems and Computing, Brunel University, London, U.K. From November 2012 to October 2014, she was an Alexander von Humboldt research fellow at the University of DuisburgEssen, Duisburg, Germany. She is currently a professor with the College of Electrical and Information Engineering, Northeast Petroleum University, Daqing, China.

Dr. Dong's current research interests include robust control and networked control systems. She is a very active reviewer for many international journals.

Zidong Wang was born in Jiangsu, China, in 1966. He received the B.Sc. degree in mathematics in 1986 from Suzhou University, Suzhou, China, and the M.Sc. degree in applied mathematics in 1990 and the Ph.D. degree in electrical engineering in 1994, both from Nanjing University of Science and Technology, Nanjing, China.

He is currently Professor of Dynamical Systems and Computing in the Department of Computer Science, Brunel University, U.K. From 1990 to 2002, he held teaching and research appointments in universities in China, Germany and the UK. Prof. Wang's research interests include dynamical systems, signal processing, bioinformatics, control theory and applications. He has published more than 200 papers in refereed international journals. He is a holder of the Alexander von Humboldt Research Fellowship of Germany, the JSPS Research Fellowship of Japan, William Mong Visiting Research Fellowship of Hong Kong.

Prof. Wang is a Fellow of the IEEE. He is serving or has served as an Associate Editor for 12 international journals, including IEEE Transactions on Automatic Control, IEEE Transactions on Control Systems Technology, IEEE Transactions on Neural Networks, IEEE Transactions on Signal Processing, and IEEE Transactions on Systems, Man, and Cybernetics - Systems. He is also a Fellow of the Royal Statistical Society and a member of program committee for many international conferences.

Steven X. Ding received Ph.D. degree in electrical engineering from the Gerhard-Mercator University of Duisburg, Germany, in 1992. From 1992 to 1994, he was a R\&D engineer at Rheinmetall GmbH. From 1995 to 2001, he was a professor of control engineering at the University of Applied Science Lausitz in Senftenberg, Germany, and served as vice president of this university during 1998 2000. He is currently a professor of control engineering and the head of the Institute for Automatic Control and Complex Systems (AKS) at the University of Duisburg-Essen, Germany.

Prof. Ding's research interests are model-based and data-driven fault diagnosis, fault tolerant systems and 
their application in industry with a focus on automotive systems and chemical processes.

Huijun Gao received the Ph.D. degree in control science and engineering from Harbin Institute of Technology, Harbin, China, in 2005.

He was a Research Associate with the Department of Mechanical Engineering, The University of Hong Kong, from November 2003 to August 2004. From October 2005 to October 2007, he carried out his postdoctoral research with the Department of Electrical and Computer Engineering, University of Alberta, Canada. Since November 2004, he has been with Harbin Institute of Technology, where he is currently a Professor and director of the Research Institute of Intelligent Control and Systems. Dr Gao's research interests include network-based control, robust control/filter theory, time-delay systems and their engineering applications.

He is a Fellow of the IEEE, and an Associate Editor for a few international journals such as Automatica, IEEE Transactions on Industrial Electronics, IEEE Transactions on Systems Man and Cybernetics Part B: Cybernetics, IEEE Transactions on Fuzzy Systems, and IEEE Transactions on Control Systems Technology. 